\title{
Surgical Extent for Ground Glass Nodules
}

\author{
Suk Ki Cho, M.D., Ph.D. \\ Division of Thoracic Surgery, Department of Thoracic and Cardiovascular Surgery, Seoul National University Bundang Hospital, Seongnam, Korea
}

\author{
ARTICLE INFO \\ Received April 15, 2021 \\ Revised June 29, 2021 \\ Accepted July 16, 2021
}

Corresponding author

Suk Ki Cho

Tel 82-31-787-7132

Fax 82-31-787-4050

E-mail tubincho@snu.ac.kr

ORCID

https://orcid.org/0000-0002-9309-8865

\begin{abstract}
As diagnoses of small ground glass nodule (GGN)-type lung adenocarcinoma are increasing due to the increasing frequency of computed tomography (CT) screening, surgical treatment for GGN-type lung adenocarcinoma has rapidly become more common. However, the appropriate surgical extent for these lesions remains unclear; therefore, several retrospective studies have been published and prospectively randomized controlled trials are being undertaken. This article takes a closer look at each clinical study. Convincing evidence must be published on 2 issues for sublobar resection to be accepted as a standard surgical option for GGN lung adenocarcinoma. In the absence of such evidence, it is better to perform lobar resection as long as the patient has sufficient lung function. The first issue is the definition of a sufficient resection margin, and the second is whether lymph node metastasis is conclusively ruled out before surgery. An additional issue is the need for an accurate calculation of the total size and solid size on CT. Given the results of clinical studies so far, wedge resection or segmentectomy shows a good prognosis for GGNs with a total size of $2 \mathrm{~cm}$ or less. Therefore, sublobar resection will play a key role even in patients who can tolerate lobectomy.
\end{abstract}

Keywords: Lung neoplasms, Ground glass opacity, Sublobar resection, Surgical extent

\section{Introduction}

Sublobar resection did not enter widespread use as an alternative surgical extent for lung cancer due to a randomized controlled trial published in 1995 that sought to determine the efficacy of sublobar resection for primary lung cancer, but failed to confirm its efficacy and validity because it showed a higher recurrence rate [1]. In recent decades, surgical treatment for nodules showing ground glass opacity (GGO) has rapidly become more common. Nodules showing GGO (ground glass nodule, GGNs) are divided into non-solid GGNs and part-solid GGNs depending on the presence of a solid component on computed tomography (CT). The typical shape of a part-solid GGN constitutes a solid component in the center that is surrounded by a GGO, but there is also a wide variety of other forms of GGNs. Persistent GGNs are likely to be cancerous lesions. Their pathologic appearance of part-solid GGNs involves an invasive solid component, which is defined as a histological subtype other than a lepidic pattern, while the surrounding GGO shows a lepidic pattern of growth, which is defined as atypical pneumocytes lining the alveolar wall with preserved architecture [2]. Therefore, the eighth edition of the AJCC/UICC (American Joint Committee on Cancer/Union for International Cancer Control) system defined the tumor size of GGN lung adenocarcinoma as the invasive component size only [3]. GGN lung adenocarcinoma is also known to have a better prognosis than pure solid tumors with an invasive component of the same size [4]. Lung adenocarcinoma is divided into 3 subtypes according to the prognosis: (1) the lepidic subtype has the best prognosis; (2) the acinar subtype has an intermediate prognosis, and (3) the solid and micropapillary subtype has the worst prognosis [5]. Therefore, the reason why GGN lung adenocarcinoma has a relatively favorable prognosis is that most of these tumors are lepidic or acinar, whereas pure solid tumors belong to subtypes with an intermediate or poorer prognosis [6].

Japanese researchers have posed the question of which CT findings correspond to pathologically non-invasive lung cancer (i.e., cancers with no lymph node [LN] metastasis or vascular or lymphatic invasion). If CT findings 
could be identified that indicate an absence of LN metastasis or lymphovascular invasion, sublobar resection would be enough for peripheral lung adenocarcinoma. Therefore, the Lung Cancer Surgical Study Group of the Japan Clinical Oncology Group (JCOG) launched the JCOG0201 study in 2002 to investigate the validity of the defined diagnostic criteria of radiologically non-invasive lung adenocarcinoma for preoperatively predicting pathologically non-invasive cancer. The results were reported in 2011 by Suzuki et al. [7]. Radiologically non-invasive lung cancer was defined as lung adenocarcinoma with a ratio of the consolidation size to the maximum tumor diameter (consolidation to tumor ratio [CTR]) less than 0.5 on the basis of past evidence, and this cutoff was used to predict pathologically non-invasive cancer. However, their predetermined criterion was not statistically confirmed. In an exploratory analysis, they examined other cutoff values, such as a CTR of 0.25 for lung adenocarcinoma with a size of $2 \mathrm{~cm}$ or less. As a result of those findings, various clinical trials were initiated, some of which are ongoing (Fig. 1) [8]. Herein, I take a closer look at each clinical study.

\section{JCOG0804/WJOG4507L}

A study entitled "The confirmatory trial of limited surgical resection for peripheral early lung cancer defined with thoracic thin section computed tomography" was conducted to evaluate the efficacy of wide wedge resection as lung parenchyma-preserving surgery for lung cancers smaller than $2 \mathrm{~cm}$ that met the criterion of a CTR less than 0.25 [9]. Between May 2009 and April 2011, 333 patients were enrolled from 51 institutions. The primary endpoint, recurrence-free survival (RFS), was estimated for 314 patients who underwent sublobar resection (wedge resection,

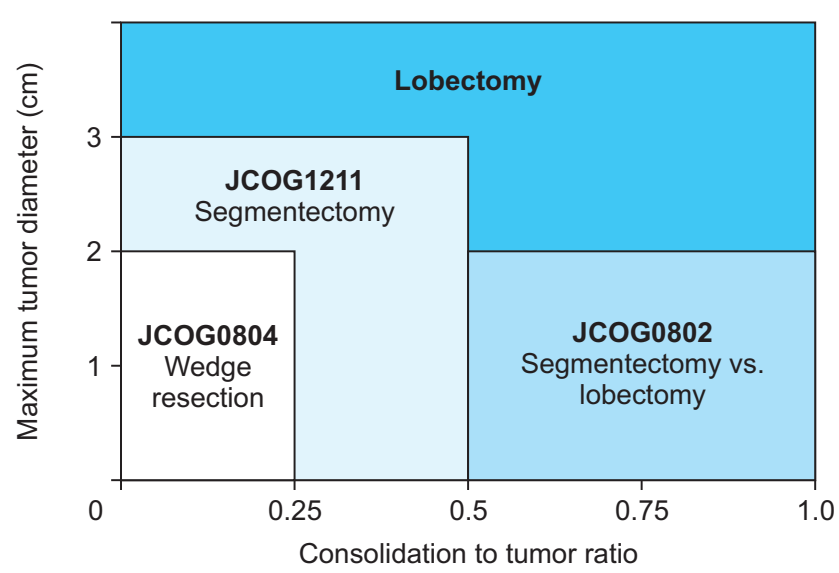

Fig. 1. Diagram of the Japan Clinical Oncology Group (JCOG) trials.
258; segmentectomy, 56). The median maximum tumor diameter on the lung window was $1.20 \mathrm{~cm}$ (range, $0.53-$ $2.00 \mathrm{~cm}$ ). The median maximum tumor diameter of consolidations was $0 \mathrm{~cm}$ (range, $0.00-0.48 \mathrm{~cm}$ ). There were no cases of local relapse. Sublobar resection, mainly wedge resection, provided sufficient local control and RFS for GGN lung cancer on CT. The authors concluded that sublobar resection should be the first choice of surgical technique if a sufficient surgical margin is preserved. To date, no clear evidence has been reported for a sufficient surgical margin during wedge resection, especially in GGN lung adenocarcinoma. The National Comprehensive Cancer Network guidelines recommend surgical margins of at least $2.0 \mathrm{~cm}$ or at least the size of the nodules.

Cho et al. [10] performed a similar study. They divided patients into 2 groups based on the CTR (CTR $\leq 0.25$, pure GGO group; CTR $>0.25$, mixed GGO group). Of a total of 97 patients, 71 were categorized into the pure GGO group and 26 into the mixed GGO group. There were 5 patients with recurrence: $1(1 / 71,1.4 \%)$ in the pure GGO group and $4(4 / 26,15.3 \%)$ in the mixed GGO group. The 5 -year overall survival rate was $98.6 \%$ in the pure GGO group and $95.5 \%$ in the mixed GGO group ( $\mathrm{p}=0.663)$. They concluded that GGO-dominant clinical stage IA lung adenocarcinoma (pure GGO group) showed an excellent prognosis. According to the authors, clinicians should carefully consider wedge resection for patients with mixed GGO nodules (CTR $>0.25$ ) because of the high recurrence rate. Radiologic noninvasiveness (CTR $\leq 0.25)$ may be a good indicator of candidates for sublobar resection in patients with early-stage lung adenocarcinoma [10]. Therefore, if this study is interpreted in a different light, more extensive resection is needed than wedge resection if a CTR $\leq 0.25$ is not clear on CT.

\section{JCOG1211}

This study was published under the title "A non-randomized confirmatory trial of segmentectomy for clinical T1N0 lung cancer with dominant GGO based on CT" in 2017 [8]. A total of 390 patients from 42 Japanese institutions were recruited within a 4 -year period. The primary endpoint of this study was 5-year RFS. Patient recruitment was finished in November 2015, and the primary analysis will be performed in 2021. This study is a pivotal trial of lung segmentectomy for early lung cancer. The results will provide clear evidence for daily clinical practice and may contribute to the preservation of pulmonary function in lung cancer patients. 


\section{JCOG0802}

This randomized controlled trial was conducted to confirm the non-inferiority of segmentectomy to lobectomy in terms of prognosis [11]. Patients with invasive peripheral non-small cell lung cancer (tumor diameter $\leq 2 \mathrm{~cm}$ ) were randomized to undergo either lobectomy or segmentectomy. The primary endpoint was overall survival. Between August 10, 2009, and October 21, 2014, 1,106 patients (lobectomy, 554; segmentectomy, 552) were enrolled. The results have not been reported, but it is anticipated that the overall survival of the segmentectomy group will not be inferior to that of the lobectomy group. Short- and intermediate-term results were published and no differences were found in almost all postoperative measures of intraoperative and postoperative complications in segmentectomy and lobectomy patients, with the exception that more air leakage was observed in the segmentectomy arm [12]. Segmentectomy will become a standard treatment if superior pulmonary function and non-inferiority in overall survival are confirmed.

A similar clinical study is now ongoing (CALGB 140503), which is a multicenter, international, non-inferiority, phase III trial in patients with peripheral non-small-cell lung cancer clinically staged as T1aN0. Patients were recruited from 69 academic and community-based institutions in Australia, Canada, and the United States. Patients were randomly assigned intraoperatively to lobar or sublobar resection. The primary endpoint of the trial is disease-free survival. Between June 15, 2007, and March 13, 2017, 697 patients were randomly allocated to lobar resection $(n=357)$ or sublobar resection ( $\mathrm{n}=340 ; 59 \%$ wedge resection).

Based on the above considerations, convincing evidence must be published on 2 issues for sublobar resection to be accepted as a standard surgical option for GGN lung adenocarcinoma. In the absence of such evidence, it is better to perform lobar resection as long as the patient has sufficient lung function. The first issue is the definition of a sufficient resection margin, and the second is whether LN metastasis is conclusively ruled out before surgery. The evaluation and dissection of LNs in GGN lung adenocarcinoma are beyond the scope of this discussion. Turning to the first issue, we are faced with the question of how wide the safety margin should be in lung cancer surgery. In general, the absolute margin should be at least $2 \mathrm{~cm}$, while in some cases where the tumor is very small, it should be equivalent to the size of the tumor [13]. Recent studies have shown that cancer cells can spread through air spaces (STAS), which means that sublobar resection may be risky even in GGN lung adenocarcinoma [14]. In other words, tumor cells can spread beyond a certain distance from in situ lesions, so lobar resection is recommended. STAS has been confirmed as the fourth mechanism of metastasis, although the presence of STAS did not affect the recurrence rate in patients who underwent lobar resection [15]. How, then, should the relationship with the visceral pleura be dealt with? Although the invasive component of a GGN may be located eccentrically, contributing to visceral pleural invasion (VPI), VPI is in fact very rare in GGN lung adenocarcinoma because the invasive component is usually surrounded by the non-cancerous portion. A recent study reported that the findings of visceral pleural indentation or attachment alone in CT predicted a poor prognosis [16]. If this result is correct, lobar resection should be performed if a GGN shows an indentation or attachment. In other words, the distance from the section cut with the stapler is important, but the relationship of the tumor with the visceral pleura must also be examined. An additional issue is the need for accurate calculations of the total size and solid size on CT. In some cases, there is a difference between CT findings and pathological findings. Such discrepancies are concerning because if the solid component of a GGN is less than $5 \mathrm{~mm}$, the best surgical extent is wedge resection, and if the solid component is more than $5 \mathrm{~mm}$, the most appropriate surgical extent is segmentectomy or lobectomy depending on the location of a tumor with an exact measured total size of $2 \mathrm{~cm}$ or less. Thus, it is very important to make accurate measurements of the total size and solid size on preoperative CT scans.

In some cases, wedge resection is performed, the tumor is diagnosed as adenocarcinoma in situ or minimally invasive adenocarcinoma based on frozen sections, and the margin is deemed sufficient, but the tumor is actually diagnosed as invasive adenocarcinoma based on permanent pathology. Furthermore, there are factors increasing the risk of recurrence risk, such as the presence of STAS. What should be done? There is no clear answer to this question, but there are 2 options: observation or additional treatment (e.g., chemotherapy, radiation therapy, or surgical resection). Some assumptions must be made to answer this question. If the patient is young and has sufficient lung function, additional treatment is likely to be more appropriate than observation, and re-operation should be strongly recommended. The major reason for recommending re-operation in such cases is that re-operation is unlikely to be difficult because not enough time would have passed since the first operation for adhesions to develop. 


\section{Conclusion}

Diagnoses of small GGN lung adenocarcinoma are increasing due to the increasing frequency of CT screening, especially in non-smoking women. Given the results of clinical studies so far, wedge resection or segmentectomy shows a good prognosis for GGNs with a total size of $2 \mathrm{~cm}$ or smaller. Therefore, sublobar resection will play a key role even in patients who can tolerate lobectomy. However, care should be taken in performing sublobar resection until the results of the ongoing JCOG0802 and CALGB140503 trials are available.

\section{Conflict of interest}

No potential conflict of interest relevant to this article was reported.

\section{ORCID}

Suk Ki Cho: https://orcid.org/0000-0002-9309-8865

\section{References}

1. Ginsberg RJ, Rubinstein LV. Randomized trial of lobectomy versus limited resection for T1 N0 non-small cell lung cancer: Lung Cancer Study Group. Ann Thorac Surg 1995;60:615-23.

2. WHO Classification of Tumours Editorial Board. WHO classification of tumours. 5th ed. Vol. 5, Thoracic tumours. Lyon: IARC Publications; 2021. 500 p.

3. Rami-Porta R, Bolejack V, Giroux DJ, et al. The IASLC lung cancer staging project: the new database to inform the eighth edition of the TNM classification of lung cancer. J Thorac Oncol 2014;9:1618-24.

4. Shin KW, Cho S, Chung JH, et al. Comparison of prognosis of solid and part-solid node-negative adenocarcinoma with the same invasive component size. Ann Thorac Surg 2017;103:1654-60.

5. Travis WD, Brambilla E, Noguchi M, et al. International Association for the Study of Lung Cancer/American Thoracic Society/European Respiratory Society international multidisciplinary classification of lung adenocarcinoma. J Thorac Oncol 2011;6:244-85.

6. Lederlin M, Puderbach M, Muley T, et al. Correlation of radio- and histomorphological pattern of pulmonary adenocarcinoma. Eur Respir J 2013;41:943-51.

7. Suzuki K, Koike T, Asakawa T, et al. A prospective radiological study of thin-section computed tomography to predict pathological noninvasiveness in peripheral clinical IA lung cancer (Japan Clinical Oncology Group 0201). J Thorac Oncol 2011;6:751-6.

8. Aokage K, Saji H, Suzuki K, et al. A non-randomized confirmatory trial of segmentectomy for clinical T1N0 lung cancer with dominant ground glass opacity based on thin-section computed tomography (JCOG1211). Gen Thorac Cardiovasc Surg 2017;65:267-72.

9. Hao D, Yang Y, Li X, Wu X, Chen Y, Wang J. Diffusion-weighted magnetic resonance imaging for predicting pathologic response to neoadjuvant chemoradiotherapy in esophageal squamous cell cancer. J Clin Oncol 2017;35(15_Suppl):e15510.

10. Cho JH, Choi YS, Kim J, Kim HK, Zo JI, Shim YM. Long-term outcomes of wedge resection for pulmonary ground-glass opacity nodules. Ann Thorac Surg 2015;99:218-22.

11. Nakamura K, Saji H, Nakajima R, et al. A phase III randomized trial of lobectomy versus limited resection for small-sized peripheral nonsmall cell lung cancer (JCOG0802/WJOG4607L). Jpn J Clin Oncol 2010;40:271-4

12. Suzuki K, Saji H, Aokage K, et al. Comparison of pulmonary segmentectomy and lobectomy: safety results of a randomized trial. J Thorac Cardiovasc Surg 2019;158:895-907.

13. Sawabata N, Maeda H, Matsumura A, Ohta M, Okumura M; Thoracic Surgery Study Group of Osaka University. Clinical implications of the margin cytology findings and margin/tumor size ratio in patients who underwent pulmonary excision for peripheral non-small cell lung cancer. Surg Today 2012;42:238-44.

14. Chae M, Jeon JH, Chung JH, et al. Prognostic significance of tumor spread through air spaces in patients with stage IA part-solid lung adenocarcinoma after sublobar resection. Lung Cancer 2021;152:216.

15. Eguchi T, Kameda K, Lu S, et al. Lobectomy is associated with better outcomes than sublobar resection in spread through air spaces (STAS)-positive T1 lung adenocarcinoma: a propensity scorematched analysis. J Thorac Oncol 2019;14:87-98.

16. Kim HJ, Cho JY, Lee YJ, et al. Clinical significance of pleural attachment and indentation of subsolid nodule lung cancer. Cancer Res Treat 2019;51:1540-8. 